\title{
EL QHaPAQ ÑAN COMO ESPACIO DE PODER DE LA POLÍTICA INCAICA
}

\author{
Reinaldo Andrés Moralejo y Diego Gobbo
}

\section{* Introducción}

Resumen

El presente trabajo se propone analizar la importancia que juega el factor de la visibilidad a lo largo del Qhapaq Ñan o Camino del Inka que atraviesa el sitio arqueológico El Shincal de Quimivil, considerado uno de los principales centros administrativos y ceremoniales del noroeste

argentino. La metodología empleada se encuentra entre una de las más potentes herramientas que ofrecen los programas SIG (Sistemas de Información Geográfica) y consistió en un análisis acumulativo de cuencas visuales, o cuenca visual acumulada (accumulated viewshed analysis). Para poder explorar el patrón de visibilidad obtenido desde

el Qhapaq Ñan se efectuó una comparación estadística entre éste y aquel de la visibilidad acumulada de un camino predictivo teórico. Los resultados logrados permiten diferenciar diversos sectores visibles y no visibles dentro del sitio, lo que parece responder a una organización espacial determinada. Esta organización podría estar pautada socialmente a través de diferentes mecanismos ideológicos y simbólicos desplegados por el Estado.

Palabras claves: Qhapaq Ñan - camino real incaico - ushnu - visibilidad Sistemas de Información Geográfica (SIG).

\begin{abstract}
This paper analyzes the importance of visibility along the Qhapaq Ñan or Inka Road that crosses the archaeological site of El Shincal de Quimivil, which is considered to be one of the main administrative and ceremonial centers of northwestern Argentina. The methodology used is one of the most powerful tools offered by GIS software and consisted of an accumulated viewshed analysis. To explore the pattern of visibility of Qhapaq Ñan, a statistical comparison was made between it and the accumulated viewshed of a theoretical predictive road. These results allowed us to differentiate visible and non-visible areas within the site that appear to be in accordance with a specific spatial organization. This organization might have been socially patterned through different ideological and symbolic mechanisms deployed by the State.
\end{abstract}

Key words: Qhapaq Ñan - Inka Road - Ushnu - visibility - Geographic Information Systems (GIS).

Recibido: Mayo 2014. Aceptado: Marzo 2015
Los caminos incaicos constituyeron un complejo sistema infraestructural compuesto por diferentes hitos culturales que respondían a las distintas necesidades del imperio inka: políticas, administrativas, ceremoniales, de comunicación, transporte y limítrofes. Para Hyslop (1984) existen cinco características principales del trazo de los caminos incaicos: la diversidad medioambiental, la diversidad cultural, las variaciones en su construcción por el régimen impuesto por los inkas en la región, la división de caminos principales y secundarios y la dispersión geográfica. Es decir, los inkas erigieron sus caminos adecuándolos al terreno y su relación con la población. En este sentido, la idea de camino inka encierra un conjunto de aspectos relacionados tanto con la geografía sobre la que se inscribe, como con aquellos elementos culturales que determinaron su específico rol en el paisaje.

De una forma u otra este sistema fue el común denominador en cada uno de los cuatro suyus en que se dividía el Tawantinsuyu. En los diversos sectores donde se ha registrado la presencia inka, nunca han faltado sus caminos: en tramos mayores o menores, en diferentes estados de conservación o con varias configuraciones adoptadas, ya sea por terrenos llanos o abruptos, han sido un elemento omnipresente en la región andina.

Los caminos constituyeron un elemento fundamental de la cultura inka; no solo estaban representados en la capital cusqueña sino que también conformaban un rasgo muy significativo en los territorios adyacentes a la misma y en aquellos lugares que el imperio fue anexando. Así, al pasar por numerosos pueblos realizaron una tarea unificadora, apoyada por la imposición de una lengua general que permitía dar unidad al incario. Una integración que fue posteriormente desestructurada por la conquista española (Rostworowsky 1988).

1 División de Arqueología, Museo de La Plata. Facultad de Ciencias Naturales y Museo, UNLP. CONICET. Av. Paseo del Bosque s/n, La Plata (1900), Provincia de Buenos Aires, ARGENTINA.Email: reinaldomoralejo@yahoo.com.ar, dgobbo@fcnym.unlp.edu.ar 
La red vial incaica tenía la particularidad de representar un complejo sistema administrativo, de transporte y comunicaciones. A través de ésta se enlazaban diversas regiones, zonas de producción con centros de consumo; se movilizaban diferentes tipos de productos, poblaciones al servicio del Estado, ejércitos, dirigentes de alto rango jerárquico, entre otras cosas (Hyslop 1984; Vitry 2000).

Además, constituía un medio en sí mismo para delimitar las cuatro divisiones (cuatripartición) del Tawantinsuyu. Según Hyslop (1992), desde el Cusco salía un camino hacia cada uno de los cuatro suyus. Este tipo de divisiones espaciales y sociales también se observaban en otros centros incaicos emplazados en las provincias; por lo que podían existir vías de entrada y salida, u otras, que dividían a la población en grupos de personas con diferentes roles y status.

Una fuerte vinculación entre los caminos y la organización socioespacial se manifiesta en el sistema de ceques, líneas que conducían hacia diferentes lugares sagrados (Wachtel 1973; Farrington 1992; Zuidema 1995). Este sistema impregnaba a los caminos de un fuerte significado geográfico y ritual convirtiéndolos en elementos del paisaje, cuya comprensión iba más allá del simple tránsito.

El paisaje no es solo un escenario donde las prácticas y relaciones de poder entre los hombres, y entre éstos y el espacio, se ven reflejadas, sino también un ámbito donde, y a partir del cual, estas relaciones se construyen (Soja 1989). En este sentido, Thomas (2001) define el paisaje como un campo relacional producto de la experiencia y de la forma en que los sujetos perciben y experimentan su entorno; por lo tanto, no existe previo a la interacción hombre-medio. El paisaje se convierte de este modo en una experiencia corporal (Ingold 2000). A lo largo de las rutas, y a través del movimiento, la acción y la percepción, se pueden producir y reproducir diferentes conjuntos de significaciones, las cuales pueden estar marcadas o señaladas en el paisaje, estableciendo diferentes relaciones de poder. Descifrarlas es una tarea que nos compete como arqueólogos. Desde ya que no constituye un trabajo sencillo, sobre todo si consideramos que la concepción del paisaje puede variar a través del tiempo y el espacio.

El Qhapaq Nan o Camino del Inka vinculaba diferentes paisajes a lo largo de miles de kilómetros, constituyendo lo que Hyslop (1992: 21) denominó "[...] la mayor evidencia arqueológica de la prehistoria americana”. Esto nos lleva a pensar que no solo podemos entenderlo como un componente físico y de alta funcionalidad, sino también como un elemento de poder que fue marcando el rumbo de determinadas situaciones al momento de la expansión y que formó parte de un paisaje significativamente simbólico, y por ende necesario, a la hora de crear alianzas con grupos locales.

De esta manera los caminos incaicos se instituían en una verdadera arquitectura de poder, la cual, en palabras de John Murra, representaba "[...] una bandera del Estado inkaico debido a su gran visibilidad y por la forma clara con la que vinculaban al individuo con la autoridad central" (Hyslop 1992: 258).

Teniendo en cuenta la importancia social y simbólica del Qhapaq Ñan, en este artículo haremos hincapié en uno de los aspectos que, según nuestras observaciones, constituye otro elemento más de significación dentro del paisaje socialmente construido: la visibilidad. El objetivo general de este trabajo radica en analizar la importancia que juega el factor de la visibilidad a lo largo de un camino incaico. Para ello se eligió como caso de estudio el Camino del Inka o Qhapaq Nan del sitio El Shincal de Quimivil, ubicado en la provincia de Catamarca, noroeste argentino. Nuestro objetivo específico consistirá en evaluar la relación visual que existe entre el camino y el sitio incaico de El Shincal de Quimivil.

Partiendo de la hipótesis de que la organización y control de un territorio se lleva a cabo de forma colectiva, se buscará determinar las diferentes relaciones existentes entre los puntos de visibilidad a lo largo del camino y el sitio El Shincal de Quimivil. De esta manera se intentará localizar redes de visibilidad heterogénea que controlen diferentes espacios, e identificar los núcleos predominantes que ofrezcan mayor protección o control del territorio. En este sentido los análisis de visibilidad permitirán el planteamiento de hipótesis sobre cómo se concibió, organizó y desarrolló la traza del camino en el medio topográfico y su relación con la estructuración urbana del sitio. La metodología empleada para realizar nuestro análisis se basó en el uso de diversas herramientas proporcionadas por un software de Sistema de Información Geográfica (SIG). 
El artículo se encuentra organizado en diferentes secciones que irán introduciendo al lector en la temática planteada. En primer lugar, como veremos a continuación, se hará referencia a la importancia que tiene el factor de la visibilidad en el estudio del paisaje. Luego, se describirá el paisaje del sitio El Shincal de Quimivil mencionando sus principales componentes arquitectónicos con especial énfasis en el camino incaico. En las secciones siguientes se detallará el procedimiento metodológico empleado en nuestro análisis e, inmediatamente, continuaremos con la presentación de los resultados obtenidos. En esta última también se presenta el modelo aplicado para generar un Camino Teórico, junto a su respectiva cuenca visual, de modo que nos permita comparar estadísticamente con el patrón de visibilidad del Qhapaq Ñan del sitio. Finalmente, se integrarán y discutirán los datos presentados seguidos, inmediatamente, por una sección de comentarios finales.

Un aspecto importante a tener en cuenta es la escasez de estudios de visibilidad sobre caminos inkas, por lo que este artículo resulta significativo en cuanto constituye un análisis novedoso que bien podría aplicarse a cualquier rasgo prolongado y continuo a través del paisaje (p.e. murallas en sitios arqueológicos defensivos, andenes y terrazas de cultivo, vías de comunicación y transporte, tendidos eléctricos, entre otros).

\section{La visibilidad como una herramienta de explora- ción del paisaje}

Los SIG constituyen complejos programas diseñados para representar y gestionar grandes volúmenes de datos sobre ciertos aspectos del mundo y que permiten capturar, almacenar, recuperar, analizar y desplegar todo tipo de información espacial; como también procesar y generar nueva información derivada de la ya existente (Gutiérrez y Gould 1994). Es decir, los SIG son una de las principales herramientas para abordar la diversidad de la información geográfica. Su utilización registra una amplia difusión dentro de la propia geografía y dentro del conjunto de estudios y aplicaciones donde el componente espacial y territorial constituye un aspecto clave del análisis (Araneda 2002). En este sentido, si bien la utilización de los SIG no tiene como finalidad restituir el paisaje antiguo, intenta mostrar de modo simplificado la complejidad de una realidad espacial con el objetivo de poder reflexionar sobre los fenómenos observados (Bartoncello y Nuninger 2002). De allí la importancia de los correspondientes trabajos en el terreno para la contrastación empírica de dichos modelos.

Uno de los análisis que ofrecen los SIG a la arqueología son los cálculos de visibilidad. Se trata de operaciones que si quisiéramos realizarlas manualmente resultarían muy costosas, y quizás ineficientes.

La visibilidad en arqueología es un tema que se viene tratando con anterioridad a la aparición de los medios informáticos. En los primeros trabajos efectuados en la década de 1970 la cuantificación de la visibilidad era tratada como una variable o atributo más del medio ambiente. Posteriormente, década de 1980 y principios de 1990, fueron evolucionando en conjunto con la teoría arqueológica, despertando gran interés dentro de los enfoques postestructuralistas (Wheatley y Gillings 2000, 2002). Su estudio, aún con muchos aspectos a mejorar, se encuentra entre una de las más potentes herramientas que ofrecen los SIG a la arqueología actual (Zamora Merchán 2013). En la siguiente sección de Metodología, haremos referencia a los diferentes factores que inciden en la determinación de la visibilidad o cuenca visual.

En cuanto al análisis de las condiciones de visibilidad en los caminos, ésta implica primeramente un conocimiento profundo de la topografía sobre la cual se desarrolla su traza. En este sentido, vale mencionar aquí una experimentación realizada por Vitry (2000) en el camino incaico Morohuasi-Incahuasi, ubicado en el Departamento de Rosario de Lerma, provincia de Salta, noroeste argentino. En la misma revela la intervisibilidad entre diferentes estructuras relacionadas directamente con el camino. Cada una de ellas fue reconocida como un sitio de comunicación visual y/o control (puestos de observación). El autor establece que estos sitios presentan una continuidad en el contexto arqueológico del tramo Morohuasi-Incahuasi, cuyo potencial de comunicación visual se complementa con la presencia de todos los demás sitios allí presentes. Asimismo propone que estos sitios pudieron cumplir un rol vinculado a la vigilancia y el control de dicho tramo (Vitry 2000).

Posteriormente, gracias al conjunto de herramientas que brindan los SIG, se pueden realizar diversos análisis es- 
paciales combinando múltiples variables, difíciles de controlar sobre el terreno. A partir de allí podremos generar un modelo de cuenca visual que nos permitirá representar, y al mismo tiempo evaluar, la percepción o dominio visual de cualquier punto natural o artificial del paisaje.

Vemos entonces que tanto los estudios sobre el terreno como los análisis SIG se pueden complementar para comprender el paisaje visual de un lugar determinado. De acuerdo con Llobera (2003) la noción de paisaje visual hace referencia a la representación visual generada por (o asociada con) una configuración espacial específica. De este modo, el paisaje visual estaría reflejando una forma de interacción social producto de diferentes relaciones de poder. En este trabajo se analizará el paisaje visual de El Shincal de Quimivil a partir del tamaño y, principalmente, la forma que toma la cuenca de visibilidad acumulada desde un elemento tan significativo como el Qhapaq Ñan.

Entendemos la visibilidad como una "forma de exhibir y destacar los productos de Cultura Material que reflejan la existencia de un grupo social" (Criado Boado 1991: 23). Esto responde a diferentes regularidades en la estrategia social de apropiación del espacio y construcción del paisaje cultural, según sea la actitud del hombre frente a la naturaleza. De acuerdo con la noción de paisaje visual, dicha estrategia estará relacionada con la voluntad de visibilidad, es decir, de la intención de hacer más, menos o nada evidente los productos de la acción social. Esta actitud particular hacia el entorno puede ser: a) de carácter inhibidor, cuando no existe un interés consciente en destacar u ocultar la presencia de la acción social o de sus resultados; b) de ocultación u ocultamiento, mediante la cual se pretende invisibilizar de manera consciente o voluntaria la acción social; c) de exhibición, cuando existe una voluntad consciente de exhibir espacialmente la naturaleza de los productos sociales; d) de monumentalización, cuando prevalece una estrategia de exhibición pero, a diferencia de la anterior, se caracteriza porque los resultados o procesos sociales perduran visualmente en el tiempo (Criado Boado 1993).

Gillings y Wheatley (2001) consideran que la visibilidad juega un rol importante en la estructuración de los paisajes arqueológicos, de allí la importancia de su análisis e interpretación. Según ellos, las características visuales de un sitio pueden ser de carácter focalizado, o deberse a su posición dentro de un paisaje visual más amplio, en el cual puede haber relaciones de visibilidad con otros sitios contemporáneos, o con componentes naturales del paisaje local y/o regional. Estos autores consideran que el termino visibilidad hace referencia a los actos cognitivos del pasado que sirven no solamente para informar acerca de la estructura y organización de su localización, sino también de las prácticas coreográficas relacionadas con ellos (Wheatley y Gillings 2000). En este sentido, de acuerdo con los enfoques recientes basados en las condiciones de visibilidad, el contexto espacial correspondería al paisaje habitado.

\section{El Qhapaq Ñan del sitio El Shincal de Quimivil}

El Shincal de Quimivil se encuentra ubicado en la localidad de Londres, Departamento de Belén, en el centro oeste de la provincia de Catamarca, noroeste argentino. Este sitio fue planificado como un centro político, administrativo y ceremonial cuyas características lo ubican entre una de las principales capitales provinciales o huamani inka del noroeste argentino (Raffino 1991, 2004). En relación a esto, se ha considerado que El Shincal de Quimivil constituye un lugar que replicaría simbólicamente la capital del imperio incaico, razón por la cual le ha sido conferida la denominación de "Nuevo Cusco" (Farrington 1998, 1999).

El origen de su emplazamiento en esta región austral del Tawantinsuyu, conocida como Kollasuyu, responde a determinados objetivos del proyecto político de expansión incaica iniciado por Pachakute (1438-1471) y proseguido por su descendencia real Topa Inka (1471-1495), Wayna Capac (1495-1525) y Atahualpa y Waskar (1525-1532). Entre estos objetivos primaba, por un lado, el interés por los recursos mineros y agropastoriles producto de una organización económica muy disciplinada basada en un sistema tributario de redistribución y reciprocidad (Raffino et al. 1978; González 1980; Raffino 1981); y, por otro, la conquista simbólica a través de la hospitalidad ceremonial reflejada en la transmisión e imposición de diversos elementos del paisaje y la cultura material (Acuto 1999, 2012; Nielsen y Walkers 1999; D’Altroy et al. 2000; Bray 2003, 2004; Williams et al. 2005).

Desde el punto de vista geográfico, El Shincal de Quimi- 


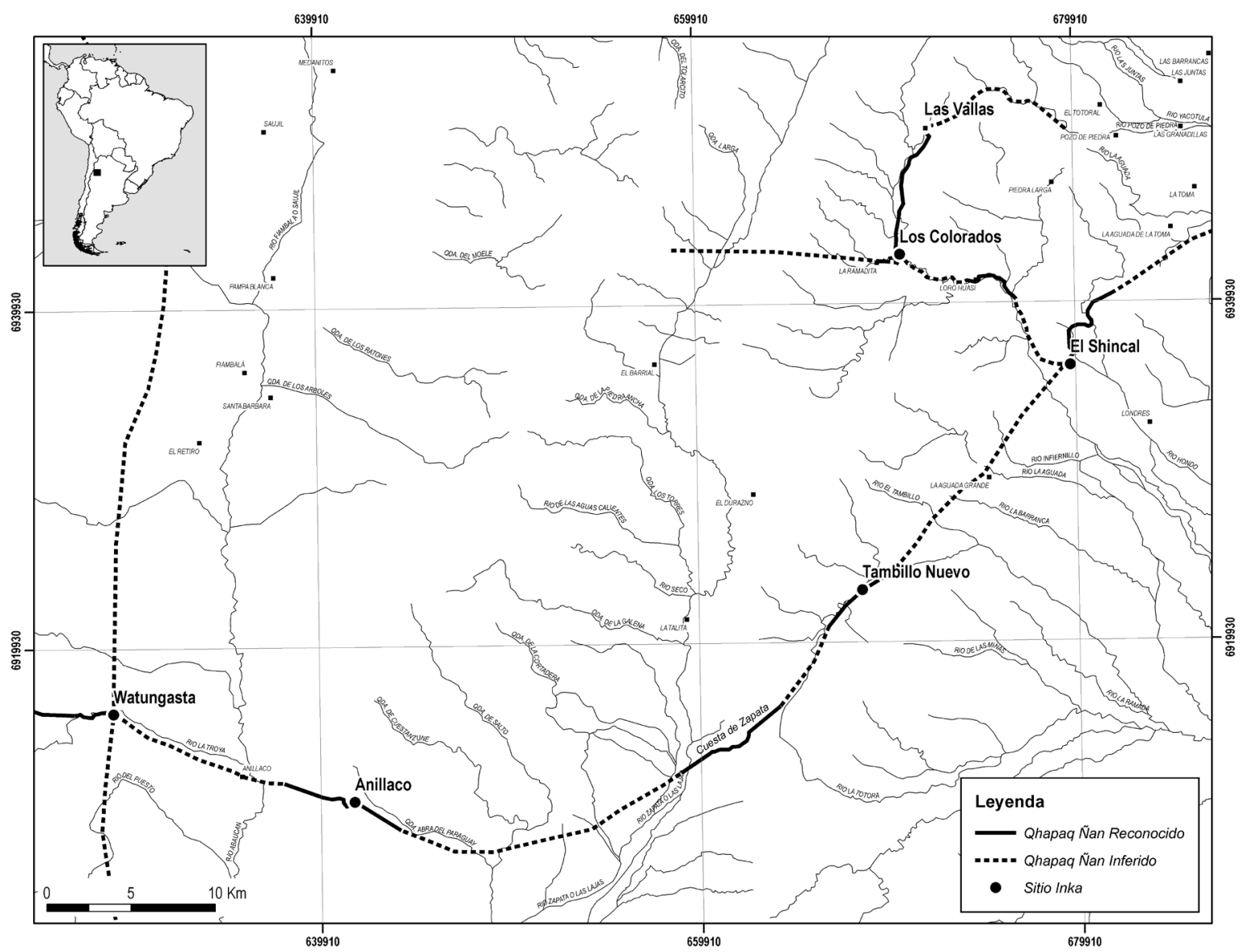

Figura 1. Red vial incaica al sur del valle de Hualfín y norte de la sierra de Zapata (proyección UTM 19S, Datum WGS84).

vil fue construido sobre el piedemonte del cerro Shincal, entre los ríos Quimivil y Hondo. Esta ubicación, conocida dentro de la cosmovisión inka como tinkuy o lugar de reunión, le ha conferido un carácter sumamente sagrado dentro del paisaje regional. Asimismo, el sitio constituyó el centro de una red caminera que comunicaba el valle de Hualfín con los valles vecinos de Abaucán o Fiambalá, El Cajón, Santa María y Bolsón de Andalgalá; situación que generó una dinámica sociocultural muy intensa durante la ocupación inka de la región (Raffino 2004; Moralejo 2011) (Figura 1).

La planta urbana del sitio está conformada por varias estructuras arquitectónicas de diferente funcionalidad, ordenadas de acuerdo a un patrón ortogonal típicamente cusqueño. Estas estructuras se encuentran distribuidas en una superficie que supera las 30 hectáreas. Entre ellas podemos mencionar: 1) una plaza o aukaipata de 175 x 175 metros, en cuyo centro se encuentra el ushnu o plataforma ceremonial; 2) dos cerros aterrazados, uno al oriente y otro al occidente de la aukaipata; 3 ) cinco grandes edificios rectangulares - kallanka-, una de ellas (K2) ubicada en el interior de la plaza paralela a su muro meridional, y las otras cuatro ubicadas por fuera de la misma, hacia el oriente ( $\mathrm{K}_{3}$ y $\mathrm{K}_{4}$ ) y occidente ( $\mathrm{K}_{1}$ y $\mathrm{K}_{5}$ ) conformando los conjuntos 5 a y 5 b, respectivamente; 4 ) acueductos de piedra que abastecían de agua al sitio; 5) estructuras rectangulares idénticas, emplazadas sobre una colina aplanada ubicada hacia el suroeste de la aukaipata. Este conjunto al comienzo de las investigaciones recibió el nombre de Atalaya. Según Farrington (2013), se trata de un patrón que replica una forma característica del área cusqueña y podría tratase de un templo u otro lugar sagrado; 6) un tramo del Qhapaq Ñan o Camino del Inka, sobre el que se centra este artículo y que será luego descripto; 7) un conjunto de unidades de almacenamiento o depósi- 
tos llamados qollqas; 8) varios conjuntos residenciales con un formato regular conocidos como kancha o RPC (Rectángulo Perimetral Compuesto), dispuestos en la proximidad de la plaza y a la vera del Camino del Inka; 9) un conjunto residencial de varios recintos denominado sinchiwasi; 10) un sector de construcciones residenciales, de mayor jerarquía, denominado Casa del Curaca o sector "Alvis". Este conjunto también responde a una variedad de kancha o RPC, y se encuentra localizado de modo aislado hacia el oeste de la plaza del sitio; 11) un gnomon o intihuatana ubicado en el cerro del Intihuatana (Figura 2).

El Qhapaq Ñan recorre el sitio de suroeste a noreste sobre el piedemonte oriental del cerro Shincal, sobre una cota que levemente supera a la del sitio (véase Figura 7). Su traza corresponde al trayecto El Shincal de Quimivil-La Aguada del Norte Chico del Departamento de Belén que

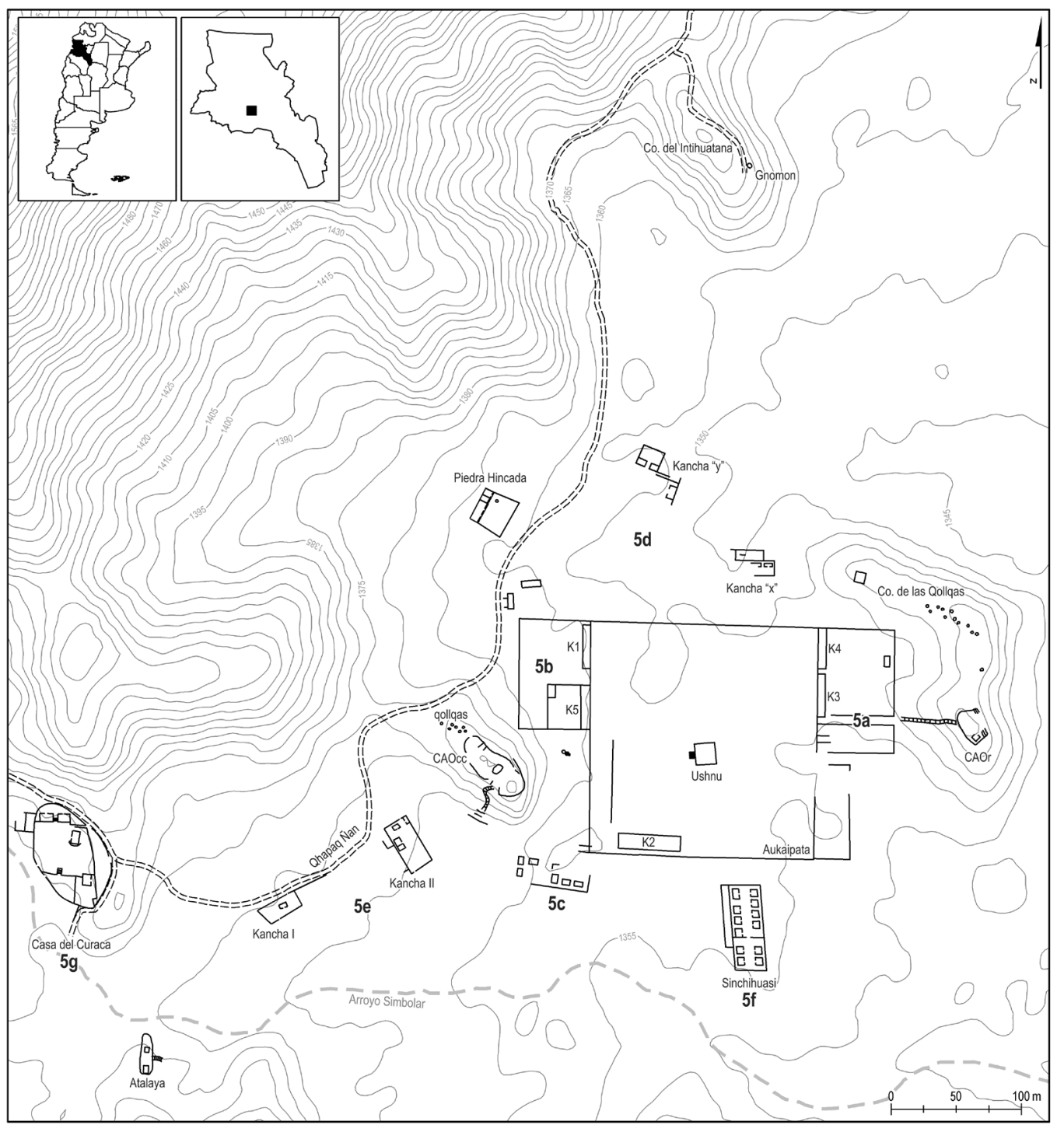

Figura 2. Plano del sitio El Shincal de Quimivil (CAOcc = Cerro Aterrazado Occidental; $\mathrm{CAOr}=$ Cerro Aterrazado Oriental) (Tomado y modificado de Raffino et al. 1982, lámina 4 y Farrington 1999: 62). 
ha sido analizada en profundidad por Moralejo (2011). A lo largo del camino se puede observar la modelización antrópica del paisaje como producto de un capital cultural y simbólico fuertemente arraigado. Entre los principales rasgos formales de este camino se han constatado en el terreno la presencia de muros de contención o retención, alineamientos contiguos de rocas, bordillos de grava, excavaciones sobre la ladera superior, muros de protección y presencia de canales para el drenaje de agua. Entre los principales elementos asociados se encuentran una serie de kancha o espacios residenciales (Casa del Curaca, Kancha I, Kancha II, Piedra Hincada y Kancha " $y$ "), un sector de estructuras de almacenamiento o qollqas, y diferentes elementos sagrados de la cosmovisión Andina como una intihuatana, o gnomon, pacchas, puquios, canales, esculturas en piedra, wankas o tokankas (Moralejo 2011).

\section{* Metodología}

Como ya dijimos, los estudios de visibilidad se encuentran entre los más importantes que ofrecen los SIG a la arqueología actual. El software SIG permitió el manejo integral de los datos espaciales recopilados, asociando datos alfanuméricos (nombres, coordenadas, descripción, entre otros) y toda la información geográfica específica de cada sitio arqueológico o hallazgo realizado durante las prospecciones arqueológicas. Se confeccionó, por lo tanto, una base de datos con varias capas temáticas desde la cual pudimos obtener algunas de nuestras interpretaciones sobre el paisaje arqueológico del área.

El método específico aplicado en este trabajo consistió en el análisis acumulativo de cuencas visuales, o de visibilidad acumulada (accumulated viewshed analysis) (Wheatley 1995). Antes de explicar de qué se trata es necesario introducir el concepto de cuenca visual o cuenca de visibilidad. Con éste nos referimos a la superficie de un territorio que es visible, o no visible, desde un punto de observación específico, a partir de una distancia determinada de visión, y en base a su topografía. Para realizar un análisis SIG de cuencas visuales se utiliza comúnmente un Modelo Digital del Terreno (MDT) de tipo raster, de manera que mediante el cálculo se pueda definir cuáles celdas son visibles (valor 1), y cuáles invisibles (valor o), desde el punto de observación especifico (Wheatley y Gillings 2002). Además, se pueden añadir otros parámetros, como por ejemplo la altura del individuo parado sobre el punto de observación. Sobre esto último volveremos cuando hagamos referencia explícita al análisis de visibilidad acumulada.

La determinación de la cuenca de visibilidad va a estar en relación con diversos factores: a) físicos, como las propiedades de la atmósfera, la cantidad y distribución de la luz, las características de los objetos que miramos, las propiedades del ojo que mira, la topografía (incluye las modificaciones debido a grandes obras públicas), la cobertura vegetal (inclusive su variación estacional) y los límites del área de estudio; y b) culturales, como el interés selectivo por los objetivos visuales, las posibilidades de desplazarse cuando se mira, alcanzar posiciones más elevadas para observar, las emociones presentes y la organización cultural del espacio (Middleton 1952; García Sanjuán et al. 2006; Zamora Merchán 2006).

En nuestro caso particular, como veremos a continuación, hemos utilizado un Modelo Digital de Elevación (DEM, por su conocida denominación en inglés Digital Elevation Model) que consiste en una representación de las elevaciones sobre un terreno (topografía), incluyendo, a diferencia del MDT, la cobertura vegetal y la arquitectura del sitio. En cuanto a la cobertura vegetal, contamos con información relativa al paleoambiente de El Shincal de Quimivil, la cual indica que, en términos generales, tanto el clima como la vegetación no han variado en los últimos 1000 años (Capparelli 1997). De todos modos, dado el tamaño de la vegetación en relación a la resolución espacial del DEM, ésta no llega a apreciarse. Lo mismo sucede con la arquitectura del sitio. Esta situación podría salvarse en la medida que logremos obtener, en el futuro inmediato, un DEM de mayor resolución a través de vuelos fotogramétricos realizados a baja altura.

Otros factores que tienen alta incidencia en el campo visual son aquellos que determinan el comportamiento de la atmósfera según la época del año y los momentos del día. En nuestra investigación, y dada la escasa distancia existente entre el Qhapaq Ñan y el sitio (la misma no supera los $4 \mathrm{~km}$ entre sus puntos más alejados), los estudios de campo muestran que la transparencia atmosférica no constituye actualmente un factor obstaculizador de la visibilidad. Si bien no contamos con datos acerca del comportamiento de la atmósfera para el momento de ocupación incaica, los estudios paleoclimáticos estarían 
revelando que la transparencia del aire pudo haber sido semejante a la actual.

En cuanto al límite de visibilidad humana se estableció un umbral de $4 \mathrm{~km}$ de radio desde cada punto de observación. Este valor de visibilidad óptima se tuvo en cuenta a partir del alcance visual obtenido desde la traza del Qhapaq Ñan durante nuestros trabajos de campo. Ello no implica que la visibilidad no sea aún mayor ya que, considerando las características del área de estudio y la cota relativa de la traza del Qhapaq Nan (mayor en relación a la del sitio), la visibilidad potencial del área se incrementa significativamente. Además, cabe comentar que este umbral de visión es coherente con el valor usado en otras investigaciones que han empleado el análisis de visibilidad (García Sanjuán et al. 2006; Cohen 2014).

Dado este conjunto de factores que condicionan la visibilidad humana siempre es recomendable, cualquiera sea el análisis que se realice, hablar de modelo de visibilidad teórico, o análisis de cuenca visual teórica o potencial (Wheatley y Gillings 2002).

Ahora, desarrollaremos el análisis específico aplicado en esta investigación y que ha sido denominado, tal como se dijo anteriormente, análisis de visibilidad acumulada. Éste se basa en la conjunción de mapas individuales de cuencas visuales de forma que, al ser sumados, se obtenga una nueva cobertura raster donde el valor de cada celda expresa la cantidad de veces que la misma es visible. Este método es conocido como método times seen (veces visto) (García Sanjuán et al. 2006) (Figura 3). Es decir, mediante este análisis obtendremos un panorama de las condiciones de visibilidad (todo aquello que es visible o no visible) del sitio El Shincal de Quimivil visto desde un elemento arqueológico determinado como, en este caso, el camino incaico.

En cuanto a la cartografía digital utilizada para la confección del SIG y la realización de los cálculos, ésta estuvo basada en:

A. Fotografías aéreas del Instituto Geográfico Nacional (IGN): fotogramas blanco y negro con una resolución máxima de 10 micrones (2540 DPI), y escala aproximada 1:75.000. Año de vuelo: 1982. Dichas imágenes fueron ortorectificadas y georeferenciadas con puntos de control en el terreno.
B. Modelo Digital de Elevación (DEM) de 10 metros de resolución espacial realizado a partir de la restitución fotogramétrica de un par estereoscópico de fotos aéreas (IGN 2C-341-0693-82-607 / IGN 2C-3410694-82-607).

C. Track del Qhapaq Ñan reconocido correspondiente al trayecto El Shincal de Quimivil - La Aguada del Norte Chico del Departamento de Belén, tomado en el terreno con GPS (Garmin GPSmap 76CSx).

D. Plano de El Shincal de Quimivil, levantado con estación total, en formato vectorial.

E. Cobertura vegetal en formato vectorial digitalizado a partir de Capparelli (1997) y ajustado y corregido a través de una clasificación supervisada sobre la fotografía aérea (Capparelli 2014, com. pers.). La altura de esta cobertura se encuentra incluida en el DEM restituido, por lo que estaría incluida en el análisis de visibilidad, pero debido al tamaño de los especímenes vegetales y la resolución del DEM no llega a apreciarse.

F. El sistema de proyección seleccionado fue GaussKrüger Argentina Faja 3, Datum WGS 84. El software utilizado para los análisis fue el ArcMap 10.1 de ESRI.

En primer lugar, para realizar el análisis de visibilidad, la traza del camino de 5460 metros de longitud fue transformada en puntos equidistantes cada 10 metros, obteniendo un total de 546 puntos. Los mismos fueron considerados como los puntos de observador para el cómputo de la visibilidad acumulada. Para tener en cuenta la altura del individuo transeúnte del camino, es decir del observador, se le agregó a cada punto una altura de 1,6 metros, correspondiente a la estatura promedio de las poblaciones andinas según Steggerda (1950).

Una vez determinada la cuenca visual para cada uno de los 546 puntos (recordemos que de cada punto se consideró un umbral de visión de $4 \mathrm{~km}$ de radio), se sumaron todos los cálculos de visibilidad (en total 546) creando un mapa de visibilidad acumulada (accumulated viewshed) desde el tramo analizado. El análisis de visibilidad acumulada con dichos puntos nos arroja una imagen raster del sitio El Shincal, donde cada pixel tiene como valor las veces que es visible, o no visible, desde el Qhapaq Ñan (recordemos que, según la resolución del DEM, cada pixel correspondería a un sector de 10 x 10 metros). De esta 

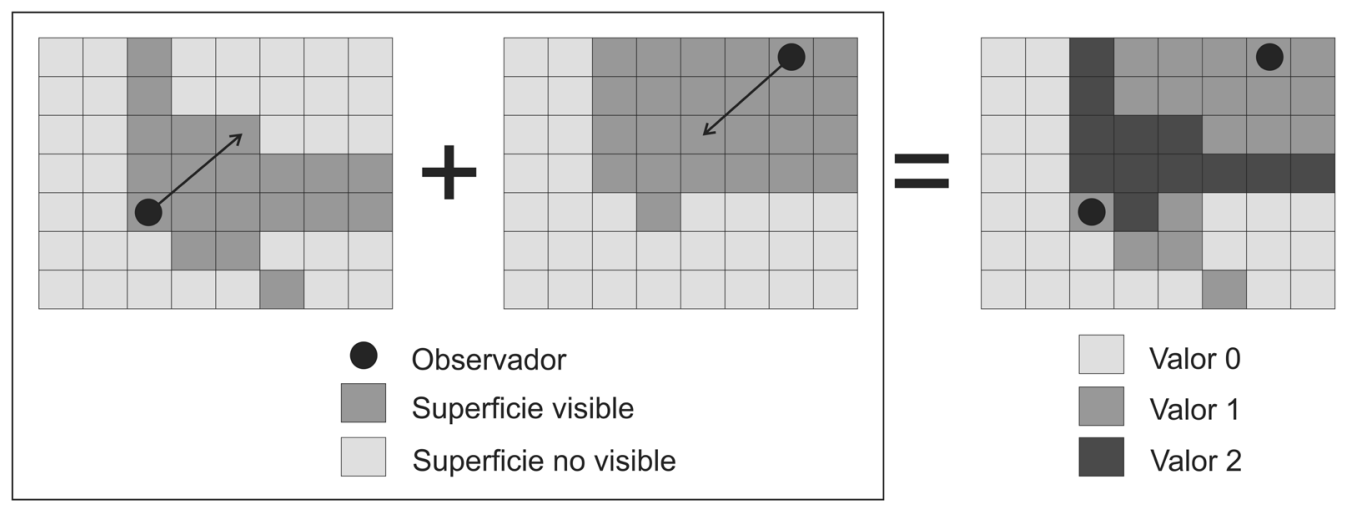

Figura 3. Representación del concepto de cuenca visual acumulada (Tomado y redibujado de García Sanjuán et al. 2006: 185, figura 2).

manera, se pueden ver los lugares de alta, baja y nula visibilidad, identificados por los diferentes matices presentes en el mapa. A través de este análisis ya se puede tener una idea integrada del paisaje visual del sitio y evaluar el grado de protagonismo que posee contemplado desde el Qhapaq Ñan.

En cuanto a la obstrucción visual que la vegetación puede ocasionar, si bien fue considerada al realizar el DEM desde la fotografía aérea, no se puede hacer evidente debido a la resolución utilizada.

Posteriormente, para poder explorar el patrón de visibilidad obtenido desde el Qhapaq Nan se efectuó una comparación estadística con la visibilidad obtenida a partir de un camino predictivo teórico. Se utilizó un camino predictivo ya que no contamos con otro camino in situ que nos permita realizar tal comparación. Las distribuciones de celdas que constituyen la cuenca visual de cada camino fueron comparadas mediante una Prueba de Bondad de Ajuste de Chi Cuadrado. La hipótesis nula sostiene que no existe diferencia significativa entre las distribuciones de visibilidad acumulada desde el Qhapaq Ñan y el camino predictivo.

El camino predictivo corresponde a un modelo teórico que se generó a través del SIG y donde se utilizaron diversas variables geográficas como factores determinantes de las trazas de los caminos (Moralejo 2011). En nuestro caso las variables utilizadas fueron la pendiente (obtenida con el DEM), la red hidrográfica (también obtenida con el DEM y mejorada digitalizando los cauces sobre las fotografías aéreas), cobertura vegetal y estructuras arquitectónicas del sitio.

Para realizar todos estos pasos de manera automática y poder utilizarlo con otros puntos y sectores, se confeccionó un modelo en el ArcMap 10.1 utilizando la aplicación ModelBuilder. Se trata de una aplicación que se utiliza para crear, editar y administrar modelos. Los modelos son flujos de trabajo que encadenan secuencias de herramientas de geoprocesamiento y suministran la salida de una herramienta a otra herramienta como entrada. ModelBuilder también se puede considerar un lenguaje de programación visual para crear flujos de trabajo (ArcGis Resources Center 2013) (Figura 4).

\section{* Resultados}

Antes de mostrar los resultados de nuestro análisis es importante señalar que, dada las características del paisaje y la cota superior de la traza del Qhapaq Ñan en relación al sitio, la visualización desde el camino supera la distancia establecida de $4 \mathrm{~km}$. La misma alcanza los abanicos aluviales de los ríos Quimivil y Hondo, sierras de Belén y el Bolsón de Andalgalá-Belén-Pipanaco. Esto lleva a obtener una perspectiva general de claro dominio visual sobre el cono aluvial del río Quimivil y el cauce del río Hondo, extendiéndose hacia la entrada de la quebrada del río El Tambillo. Cabe destacar también el dominio visual sobre el sector pedemontano, ocupado por el bosque abierto con arbustal espinoso caducifolio. 


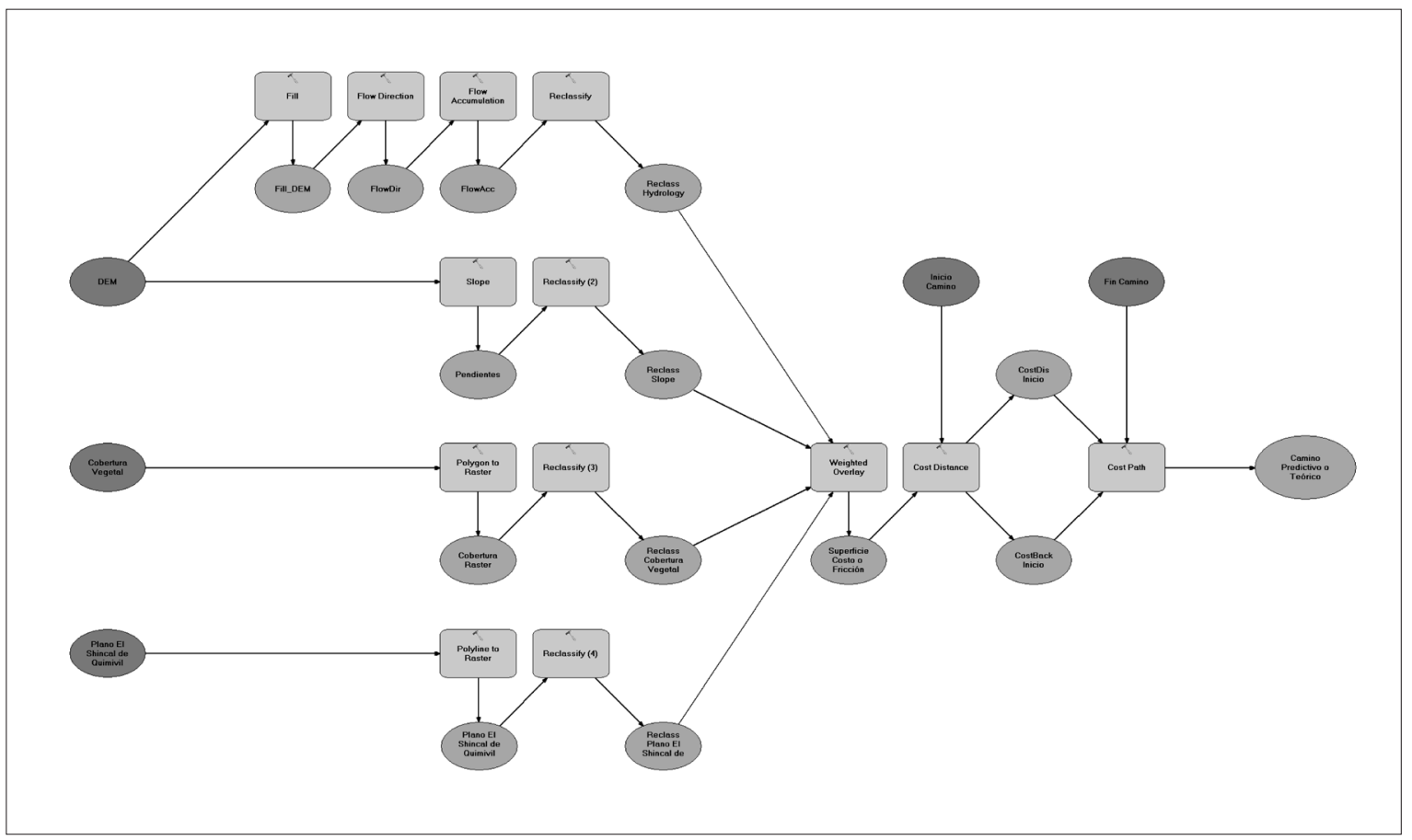

Figura 4. Modelo elaborado a partir de la aplicación ModelBuilder.

A continuación, nos concentramos en el análisis de visibilidad acumulada realizado sobre el sitio El Shincal de Quimivil, teniendo en cuenta solo los límites actuales del polígono perimetral ${ }^{2}$. Allí se pueden observar sectores visibles y no visibles desde el Qhapaq Ñan, logrando de este modo una primera aproximación a nuestro objetivo principal (Figura 5). Cuando hablamos de lo visible nos referimos a sectores o lugares del sitio, de 10 por 10 metros (lo que corresponde a un pixel según la resolución utilizada), que al menos una vez son vistos desde el Qhapaq Ñan. Mientras que cuando hablamos de lo no visible se trata de sectores del sitio que no se ven desde ningún punto a lo largo del Qhapaq Ñan.

El total de pixeles del sitio es de $\mathrm{N}=32 \mathrm{O} 2$, lo que corresponde a una superficie de 32,02 hectáreas. Los sectores visibles representan el 96,16\% ( $n=3079)$, mientras que

2 El polígono perimetral del sitio ocupa una superficie aproximada de 32,02 hectáreas. En la actualidad la Dirección de Antropología de Catamarca se encuentra en un proceso gestión administrativa para la ampliación del mismo.

3 Este breve segmento de Qhapaq Ñan posee un sentido suroeste en dirección a la quebrada del río El Tambillo (González 1966; Moralejo 2011) los no visibles representan el 3,84\% $(n=123)$ del total.

Dentro del 96,16\% de sectores visibles se puede observar que la visibilidad sobre el sitio no se distribuye de manera homogénea. En la Figura 5, la diferenciación entre los sectores de mayor visibilidad (representados por los tonos grises claros y el blanco) y los de menor visibilidad (representados por los tonos grises oscuros) indica que: los sectores del sitio que más se ven desde el Qhapaq Ñan se encuentran ubicados hacia el oriente del sitio, comprendiendo la plaza o aukaipata, el ushnu, el sector 5a, los cerros aterrazados oriental y occidental, el cerro de las Qollqas, la Kancha "x" del sector $5 \mathrm{~d}$ y el conjunto $5 \mathrm{f} \mathrm{o}$ sinchihuasi (Figura 6); mientras que los sectores de menor visibilidad se ubican hacia el occidente del sitio comprendiendo la Casa del Curaca, el Atalaya, las kancha I y II del sector 5 e, el sector 5 b, una parte del sector 5 c y las kancha Piedra Hincada y Kancha "y" del sector $5 \mathrm{~d}$.

En cuanto al 3,84\% de sectores no visibles (tono negro), tampoco existe una distribución homogénea en el sitio. Estos se concentran al sur de la Casa del Curaca en un espacio correspondiente al Arroyo Simbolar y un breve segmento del Qhapaq Ñan?3. El resto de los sectores no vi- 


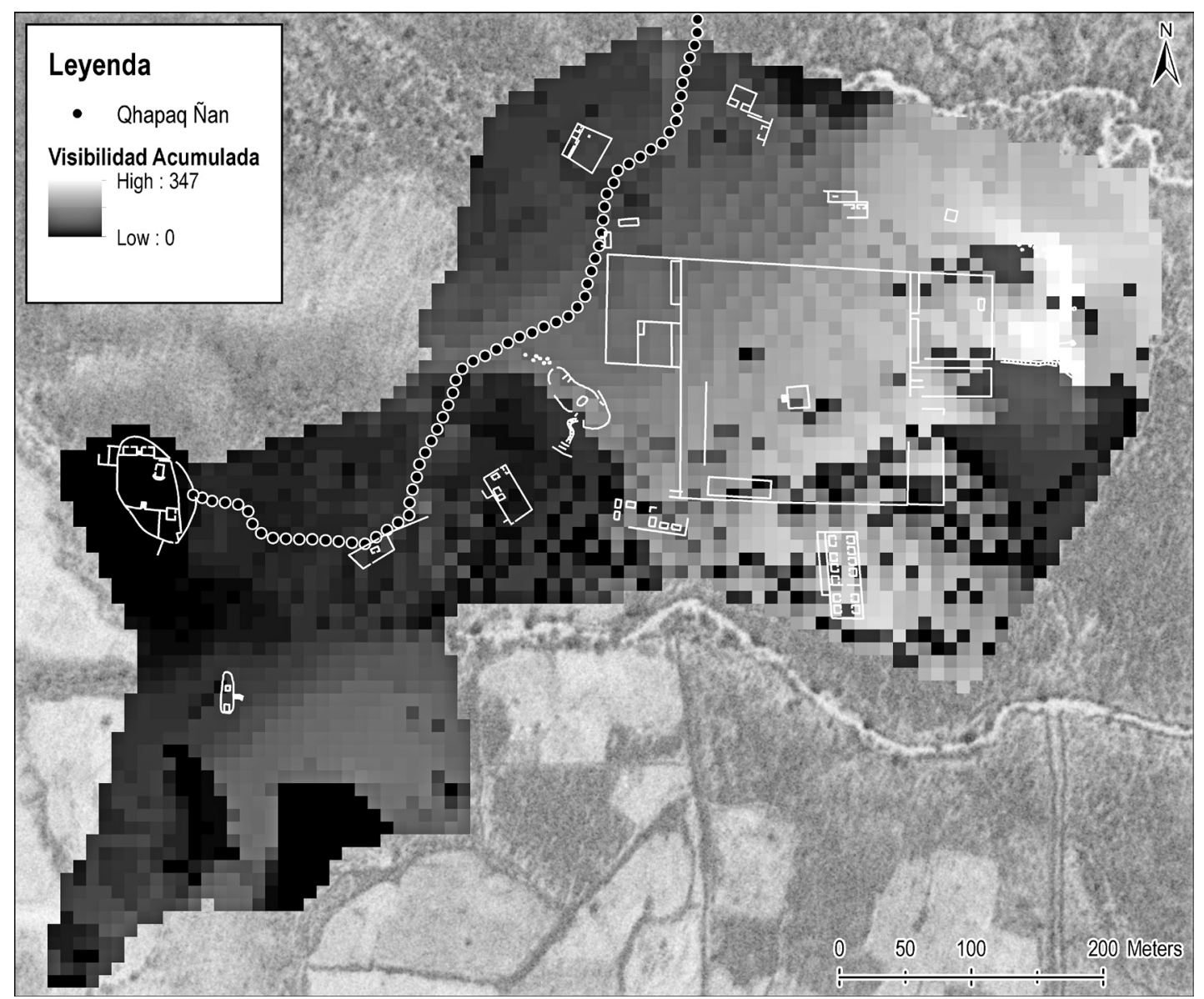

Figura 5. Mapa de visibilidad acumulada — accumulated viewshed - del sitio El Shincal de Quimivil generado desde el Qhapaq Ñan.

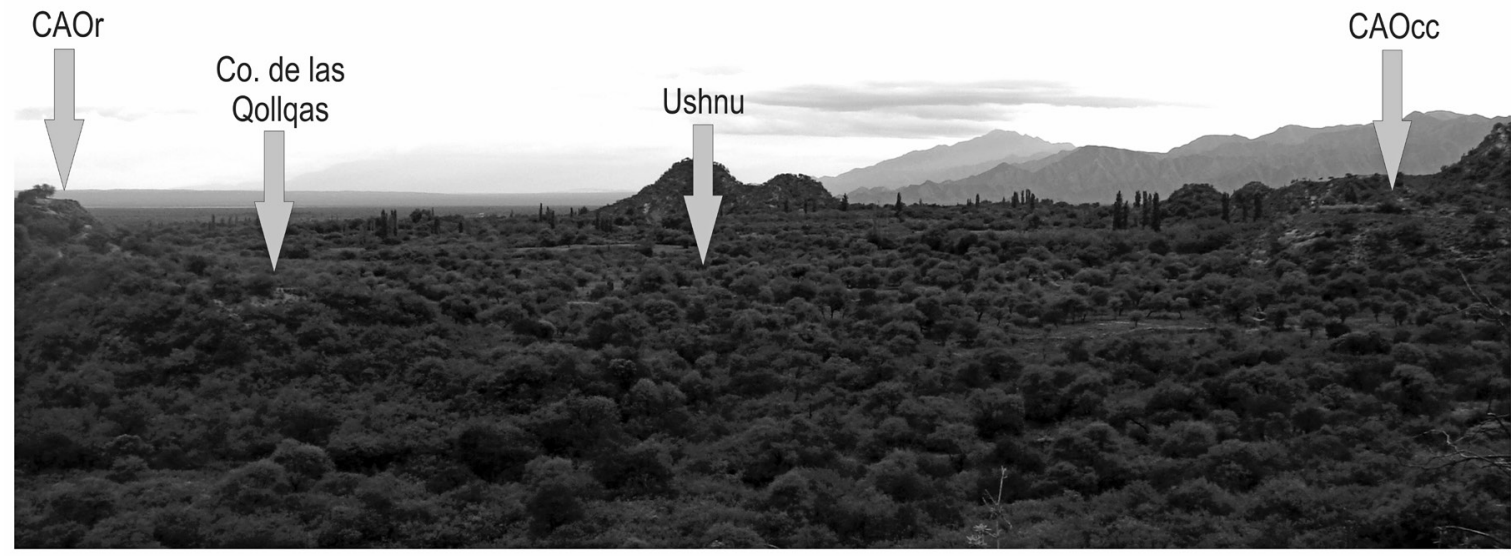

Figura 6. Perspectiva de El Shincal hacia el sur donde se muestran algunos de los sectores más visibles desde el Camino del Inka (fotografía tomada desde el cerro del Intihuatana durante una campaña en el mes de marzo de 2009). 
sibles se distribuyen mayormente hacia el suroeste (sector 5c) y este-sureste de la aukaipata.

\section{Modelización de un camino predictivo}

Para poder explorar el patrón de visibilidad obtenido desde el Qhapaq Ñan se efectuó una comparación estadística con la visibilidad acumulada obtenida a partir de un camino predictivo, o teórico. Se utilizó un camino predictivo ya que no contamos con otro camino in situ en el sitio que nos permita realizar tal comparación.

El camino predictivo constituye un modelo teórico que se construyó a través del SIG y donde se utilizaron diversas variables como factores determinantes de la traza del camino (Hyslop 1992; Moralejo 2011). La idea de construir un modelo predictivo recae sobre la posibilidad de comprender los procesos sociopolíticos que subyacen a la construcción del paisaje. Si bien esta herramienta no está libre de cuestionamientos (Kvamme 1990; Wescott 2000), su utilización no tiene como finalidad restituir el paisaje antiguo, sino más bien mostrar de modo simplificado la complejidad de una realidad espacial, con la finalidad de poder reflexionar sobre los fenómenos observados (Bartoncello y Nuninger 2002). Es decir, los modelos predictivos son herramientas para proyectar patrones o relaciones conocidas a lugares y momentos que no lo son (Warren y Asch 2000). Su aplicación se ha visto favorecida en los últimos tiempos, gracias al desarrollo de tecnologías informáticas propias de los SIG (Warren 1990; Duncan y Beckam 2000). Estos se basan en un profundo manejo de la información geográfica utilizando, entre otras cosas, programas estadísticos multivariados, programas de mapeo y cartografía digital primaria de calidad (Wheatley y Gillings 2002; De Feo y Gobbo 2006). Asimismo, la construcción del modelo predictivo requiere un profundo conocimiento arqueológico tanto del sitio en particular como del área en general, de modo que permita examinar y sopesar su validez potencial.

Para la elaboración del camino predictivo se tuvieron en cuenta un conjunto de variables que podrían condicionar la traza de cualquier vía: pendiente, red hidrográfica, cobertura vegetal y estructuras arquitectónicas del sitio. Partiendo de la cartografía primaria ya mencionada en la sección de metodología se elaboró un DEM (Digital Elevation Model) con una resolución de $10 \mathrm{~m}$. A partir de éste se obtuvieron las siguientes capas o superficies: 1) un mapa de pendientes, donde se efectuó una reclasificación de las clases de pendientes, adjudicando un valor más alto a las zonas de pendientes más abruptas; 2) un mapa de la red hidrográfica obtenido con el DEM, donde se cotejó la información generada con la interpretación visual de las fotografías aéreas. El archivo raster se reclasificó para, posteriormente, posibilitar la superposición con el mapa de pendientes; 3 ) un mapa de cobertura vegetal, donde se diferenció cada comunidad vegetal y luego se reclasificó para adjudicarles un valor de acuerdo a su grado de transitabilidad; 4) por último se utilizó el plano del sitio, donde se diferenciaron aquellas estructuras que no podían ser atravesadas por el camino.

Con dichas superficies o capas se generó un mapa de costo o fricción que representa la rugosidad del terreno en función de cada una de las variables. Luego, partiendo de las variables mencionadas, se generó a través de la función Cost-weighted (costo acumulado) del programa ArcMap 10.1, una superficie continua que expresa la fricción de desplazamiento hacia distintos puntos espaciales desde un determinado punto de partida, generalmente un sitio (o punto de interés). En nuestro caso se tomó como punto principal de partida el Abra Casa del Curaca. En esta superficie formada queda representado el terreno según el grado de resistencia o permeabilidad que ofrece para el desplazamiento humano, lo que podríamos llamar entonces un modelo general de coste de desplazamiento o modelo de circulación pedestre (Grau Mira 2006). Finalmente, con la función Cost Path (camino óptimo) del programa ArcMap 10.1, ese mismo mapa de costo o fricción sirvió para trazar el camino óptimo, entendido como la línea que cubre el trayecto entre dos puntos en función del coste mínimo de recorrido de la superficie de fricción. De esta manera se obtiene, finalmente, un modelo de camino óptimo (Grau Mira 2006). En el esquema de la Figura 4 se representa la serie de pasos seguidos en la elaboración de nuestro modelo teórico.

La traza teórica del camino predictivo, a diferencia de la traza del Qhapaq Ñan, se desarrolla en términos generales por el sector medio del sitio. Comenzando en la Casa del Curaca, la traza se dirige hacia el sureste, pasando entre las kancha I y II, atravesando luego la aukaipata en sentido suroeste a noreste y, finalmente, bordeando el sector occidental del cerro de las Qollqas en dirección ha- 


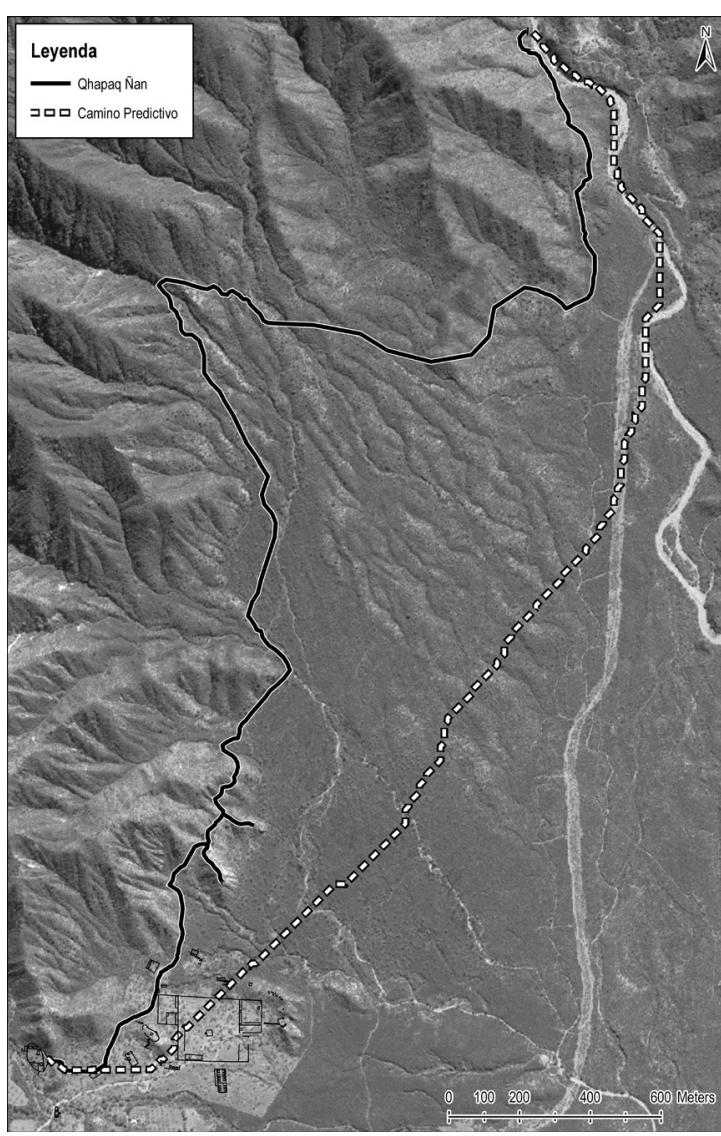

Figura 7. Comparación entre las trazas del Qhapaq Ñan (línea llena) y del camino predictivo (línea punteada).

cia el río Hondo a través del piedemonte del cerro Shincal (Figura 7).

La traza teórica del camino predictivo fue dividida en 407 puntos tomados cada 10 metros. Ya habíamos dicho que el total de pixeles del sitio es de $\mathrm{N}=3202$. De acuerdo con los resultados del modelo teórico, existen 3000 sectores visibles (lo que representa un 93,70\%) y 202 sectores no visibles (lo que representa un 6,30\%) desde el camino predictivo.

Posteriormente, a dicho modelo de camino predictivo se le aplicó el mismo examen de visibilidad acumulada - accumulated viewshed - empleado para el trazado del Qhapaq Ñan. Los resultados de este análisis indican que los sectores más visibles del sitio (representados por los tonos grises claros y el blanco) se concentran, por un lado, hacia la porción oeste de la aukaipata cubriendo el cerro Aterrazado Occidental, el sector 5b, las kancha del sector $5 \mathrm{~d}$ y el sector ubicado al sur del Atalaya; y, por otro lado, hacia el cerro Aterrazado Oriental y el cerro de las Qollqas. Por otra parte, los sectores de menor visibilidad (representados por los tonos grises oscuros) se encuentran hacia el occidente del sitio, al igual que lo observado en el análisis del Qhapaq Nan, donde yacen la Casa del Curaca, las kancha I y II del sector 5 e y el Atalaya; y hacia el oriente de la aukaipata donde yacen principalmente el sector 5 a y el conjunto 5 fo sinchihuasi (Figura 8 - abajo).

En cuanto al 6,30\% de sectores no visibles (tono negro), volvemos a destacar la no visibilidad de un sector ubicado al sur de la Casa del Curaca dominado por el arroyo Simbolar y un breve segmento del Qhapaq Ñan; y del área ubicada hacia el sureste de la aukaipata (Figura 8 - abajo).

\section{Comparación entre la visibilidad del Qhapaq Ñan y nuestro modelo de camino predictivo}

Con el propósito de determinar si el patrón de visibilidad acumulada obtenido desde el Qhapaq Ñan se ajusta al del camino predictivo se realizó una comparación estadística mediante una prueba de Bondad de Ajuste de Chi Cuadrado. La hipótesis nula sostiene que no existe diferencia significativa entre las distribuciones de visibilidad acumulada desde el Qhapaq Ñan y el camino predictivo. Es decir que la distribución de visibilidad acumulada del Qhapaq Ñan se ajusta a la distribución de visibilidad acumulada del camino predictivo. La comparación entre ambas distribuciones se realizó con un nivel de significación del o,ool.

$\mathrm{Ho}=\mathrm{Fo}=\mathrm{Fe}$

$\mathrm{H} 1=\mathrm{Fo} \neq \mathrm{Fe}$

En la Figura 8 se muestra la diferencia existente entre las distribuciones de visibilidad acumulada generada desde cada traza. En el caso del modelo teórico existe un corrimiento del control visual hacia el oeste de la aukaipata, haciendo más visible determinados sectores que el Qhapaq Ñan mostraba con menor visibilidad: el sector 5 b y las kancha Piedra Hincada y Kancha "y" del sector 5 d. Asimismo, el modelo teórico le resta visibilidad al sector 5 a y al conjunto 5 f o sinchihuasi ubicados hacia el oriente y el sur de la aukaipata, respectivamente. 
La prueba realizada arrojo una probabilidad menor al 0,0001, por lo cual nuestra hipótesis nula se rechaza ( $\mathrm{Fi}-$ gura 8). De este modo podemos decir que la distribución de frecuencias de visibilidad acumulada del Qhapaq Ñan tiene una diferencia altamente significativa con respecto a la distribución del camino predictivo. Esto permite comenzar a pensar que el lugar para emplazar la traza del Qhapaq Ñan ha sido producto de una selección consciente y controlada por partes de los inkas, deliberando cuáles sectores del sitio podían ser visibilizados y cuáles invisibilizados, independientemente del costo-beneficio del trazado.
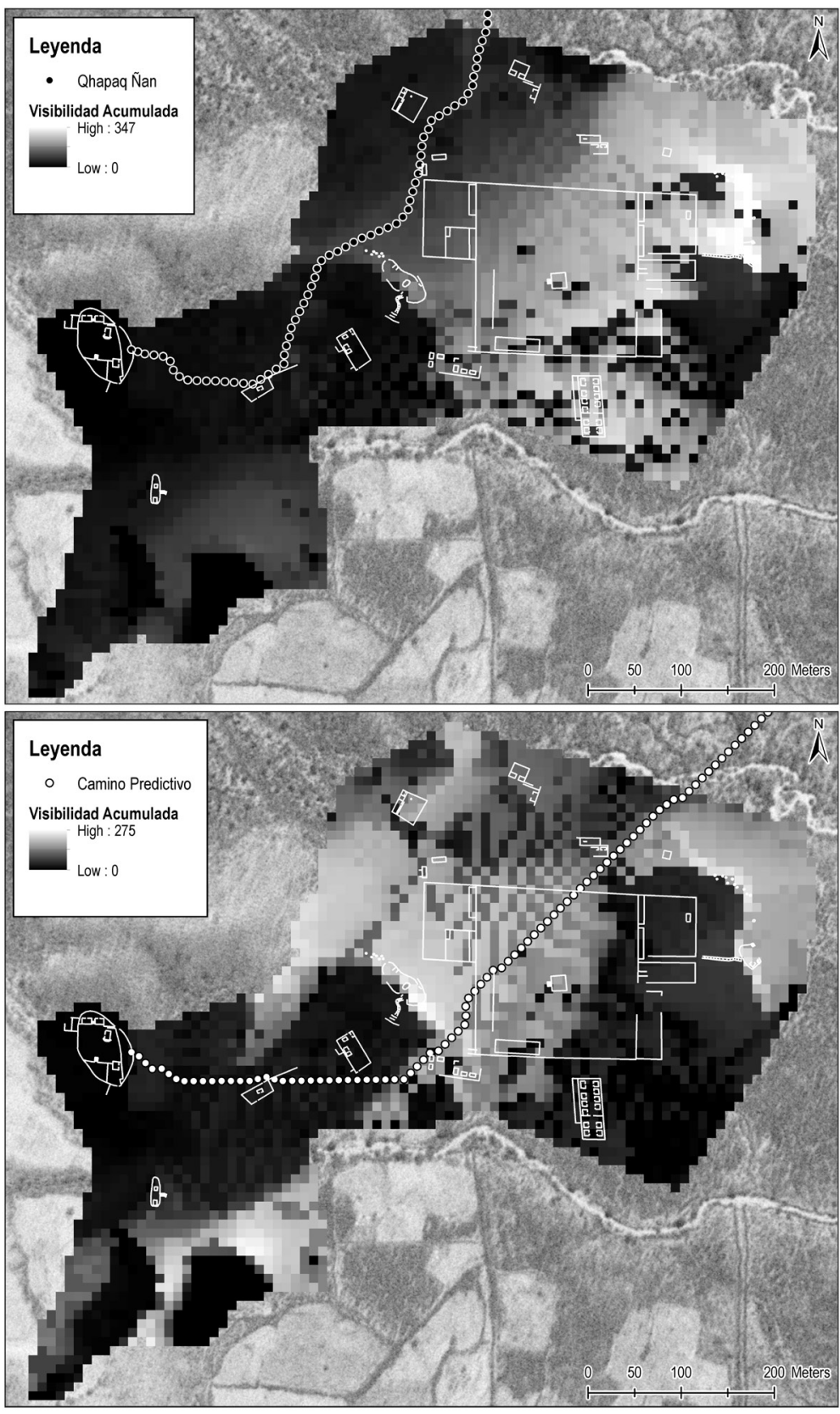

Figura 8. Comparación entre las cuencas de visibilidad acumulada del Qhapaq Ñan (arriba) y camino predictivo (abajo). La Prueba de Bondad de Ajuste de Chi cuadrado $(\mathrm{p}<0,001)$ arrojó una $\mathrm{P}<0,0001$ lo que indica una diferencia altamente significativa entre ambas distribuciones de frecuencias de visibilidad acumulada. 


\section{$\because$ Discusiones}

En este artículo hemos analizado cómo, a través de la determinación de las características visuales de un sitio, se puede comprender la modelización del paisaje arqueológico. Es decir, si existió por parte de los inkas una estrategia cultural de organización del paisaje visual que pueda explicar la forma de poder político y simbólico concebida en el sitio y la región.

Se sabe que los inkas tenían una forma particular de organizar el espacio, y en esta investigación podemos ver cómo la misma se manifiesta a través de la estructuración del sitio entre sectores con mayor, nula y escasa visibilidad. Este paisaje visual presenta entonces determinadas condiciones de visibilidad que responden a la voluntad social que tenían los inkas de hacer más, menos o nada evidente las manifestaciones formales de su sociedad y la acción social que las produjo. En este sentido, de acuerdo con nuestro análisis de visibilidad, podemos decir que en El Shincal de Quimivil existió una doble estrategia de visibilización basada, por un lado, en la exhibición/monumentalización de la aukaipata y sus componentes asociados $y$, por otro, en la ocultación de un conjunto determinado de espacios residenciales.

La estrategia de exhibición/monumentalización busca enfatizar dentro del paisaje visual determinados productos culturales cuyos efectos sociales sean significativos y perdurables en el tiempo. Se trata, nada más y nada menos, que de aquellos componentes directamente vinculados con la gran plaza, o aukaipata, como son los cerros aterrazados, los edificios kallanka (principalmente la K2 ubicada en el interior de la aukaipata) y el ushnu, y que en su conjunto representan los máximos exponentes de la arquitectura pública de poder inka. Las prácticas sociales desarrolladas en este sector serían abiertas, o públicas, vinculadas con reuniones comunales de carácter político, ceremonial y festivo. En este sentido resulta interesante el planteo de Moore (1996) donde pone de manifiesto la proxémica de las plazas incaicas con respecto a los rasgos y estructuras vecinas. Éstas se ubican generalmente en el sector central del sitio, constituyendo un elemento clave - de presencia y poder- del espacio cívico ceremonial incaico.

Por otra parte, los sectores del sitio con escasa y nula visibilidad desde el Qhapaq Ñan estaban vinculados con lugares donde se realizaban actividades restringidas ya sea domésticas y/o rituales y donde solo tenían acceso determinados grupos de personas. Respondían entonces a una estrategia de ocultación u ocultamiento, que buscaba voluntariamente invisibilizar la presencia de la acción social y sus resultados. Como parte de esta estrategia encontramos diferentes kancha incaicas, asociadas directamente al camino, como es el caso de la Piedra Hincada, Kancha "y", Kancha I, Kancha II y Casa del Curaca (Couso et al. 2011; Moralejo y Couso 2012; Moralejo 2013).

En el caso particular de la Casa del Curaca - de posición bien aislada y de difícil accesibilidad con respecto al resto del sitio-, la visibilidad restringida constituye un elemento singular que sumado a su arquitectura y restos de vasijas de excelente calidad (González 1966; Raffino 2004; Giovannetti et al. 2012) y la trifurcación de caminos (Moralejo 2011) refuerzan la idea de que constituyó una de las posibles residencias para las elites gobernantes de El Shincal de Quimivil.

Diversos trabajos, arqueológicos y etnohistóricos, acerca de la arquitectura inka a lo largo del Tawantinsuyu han demostrado esta organización del espacio que alterna entre lo público y lo restringido o privado. Dicha organización no solo se materializaba a través de la arquitectura en superficie, sino también por la presencia de determinados contextos arqueológicos registrados en excavaciones (Gasparini y Margolies 1977; Hyslop 1990; Matos Mendieta 1994; Makowsky et al. 2005; Raffino 2007; Farrington 2013; Morris 2013; Raffino et al. 2014). Si bien en este artículo se plantea y explora un nuevo indicador, como es la visibilidad acumulada, para analizar la relación entre la traza del Qhapaq Ñan y el resto del sitio - para luego tratar de comprender la heterogeneidad de la organización espacial incaica-, no pretendemos establecer ningún tipo de causalidad para su diferenciación. Por el contrario, creemos que estos conceptos acerca de lo público y lo privado conllevan una discusión amplia y compleja que debería ser revisada, problemática que por el momento escapa al objetivo de nuestro trabajo.

Lo analizado hasta aquí deja entrever entonces, que la visibilidad de El Shincal de Quimivil estaba culturalmente pautada bajo la estela de una doble estrategia de visibilización que contribuía a subrayar la monumentalidad y el ocultamiento de diferentes sectores. De este modo, el 
paisaje visual reflejaba una determinada racionalidad espacial, que permitía reproducir ciertas relaciones de poder entre los diversos actores sociales, conformando un paisaje político cargado de significado. El espacio no solo era contemplado como la matriz física de la acción humana sino también social y simbólica, y por ello, dinámica (Criado Boado 1991, 1997). Es decir, las comunidades transformaban los espacios físicos en lugares llenos de contenidos mediante sus actividades diarias, sus creencias y sus sistemas de valores (Anschuetz et al. 2001).

Ahora, hay que resaltar que esta organización espacial queda manifiesta a través de un análisis de visibilidad generado desde el Qhapaq Ñan, lo cual constituye un dato atractivo y novedoso teniendo en cuenta el rol que tenían los caminos para el Estado.

Los caminos poseían (y poseen actualmente) múltiples significados que, para el caso de los inkas, estaban relacionados con decisiones políticas de expansión, presencia, dominación y poder. Según Hyslop (1992: 31), "para los pueblos conquistados a lo largo de todo el Imperio, los caminos constituían un símbolo omnipresente del poder y autoridad del Estado Inka". De hecho los pueblos locales se encargaban de construirlos y mantenerlos con su trabajo, como parte del conjunto de obligaciones que tenían con aquél. Asimismo, además del significado práctico que los caminos pudieron alcanzar, jugaron un rol especial en conceptos vinculados con la división del espacio y la sociedad. Conceptos que no estaban exentos de un significado ritual, y que fueron explícitamente usados para comprender y expresar la geografía cultural (Hyslop 1992: 255). En este marco, el paisaje quedaba inmerso en el contexto de los sistemas de poder vigentes en la sociedad, razón por la cual la organización espacial cumplía un rol legitimador en relación a las desigualdades sociales propias de los sistemas sociales, produciendo y reproduciendo relaciones de dominación. Aquí las espacialidades podrían verse reforzadas y reproducidas, como también sujetas a cambios que llevaran a su reestructuración, tanto en su materialidad como en su significado (Bender 1993; Moore 1996; Acuto 1999; Thomas 2001).

El Qhapaq Nan de El Shincal guardaba una relación de dominio y control visual muy estrecha con el espacio urbano del sitio. Una relación que dejaba al descubierto un paisaje basado principalmente en la monumentalización de los máximos exponentes de la arquitectura pública de poder Inka. Pudo quizás, actuar a modo de panóptico como un espacio de control visual directo para obtener información de los acontecimientos que tenían lugar en la aukaipata. Es decir, desde el camino ya se transmitía un mensaje ideológico de presencia y autoridad, que permitía reproducir en el transeúnte las relaciones de dominación y poder promovidas por el Estado.

Esta relación de poder, por supuesto, no solo se manifestaba a través de la percepción visual sino también mediante otros factores que permitían definir el trazado del Qhapaq Ñan. En este sentido coincidimos con el planteo de Llobera (2007) acerca de la multisensorialidad de la experiencia espacial, por lo que es importante continuar con otros tipos de análisis (p.e. arqueoastronómicos, intervisibilidad entre diversos componentes del sitio, visibilización o forma cómo un elemento arqueológico es visto) que permitan complementar el enfoque visual y complejizar aún más la relación entre el camino y el sitio.

\section{* Comentarios finales}

El análisis de visibilidad acumulada presentado en este artículo ha servido para explorar la relación visual existente entre el Qhapaq Ñan del sitio El Shincal de Quimivil y el sitio propiamente dicho. Los resultados demuestran la existencia de un paisaje visual culturalmente pautado cuya configuración espacial estaba conscientemente señalada y quizás también, por qué no, planificada desde el camino. En este sentido, la construcción del camino debió estar condicionada no solo por la topografía circundante y la presencia de otros elementos sagrados propios de la cosmovisión Andina, sino también por el factor visibilidad. Un factor de prestigio (García Sanjuán et al. 2006) que tendía a enfatizar, por un lado, la monumentalidad del espacio cívico ceremonial de poder estatal y, por otro, el ocultamiento de determinados espacios residenciales. Esta estrategia se convertía entonces en un ejercicio permanente de apropiación del espacio y de constitución del paisaje como territorio, y que a su vez legitimaba el rol del camino como espacio de poder de la política incaica. De este modo, podemos decir que el Qhapaq Ñan fue un sistema que no estaba basado únicamente en su infraestructura, sino también constituyó "[...] una superes- 
tructura de control de los pueblos locales, una poderosa herramienta de conquista y dominación física y simbólica del área por el Inka" (Berenguer 2007: 433).

Si bien creemos que no existe una relación unívoca entre espacio y forma de organización social, este tipo de análisis mensurable y apoyado en evidencias empíricas permite diferenciar, a manera de hipótesis, los tipos de espacios con mayor, menor o nula visibilidad dentro de un sitio. Espacios que, como hemos visto, estaban pautados socialmente a partir de aspectos ideológicos y mecanismos simbólicos (Gil García 2001). Así, la visibilidad o invisibilidad de determinados espacios se convierte en un instrumento de producción de significado y control social modelado por la ideología dominante.

Como ha quedado demostrado, esta metodología — basada en la suma de diferentes áreas de visibilidad a lo largo de un camino - permite superar el resultado del cálculo de visibilidad realizado desde un único punto de observación. De acuerdo con Zamora Merchán (2013) se trata de un estudio complejo que alude al dinamismo y particularidad de los diversos contextos de la vida en los paisajes. Igualmente, vale considerar que si bien este tipo de estudios, en comparación con aquellos que se realizan desde un único punto de vista, tienden a minimizar la frecuencia de sectores no visibles, es necesario analizar el grado de accesibilidad a estos sectores a fin de profundizar más en el estudio del costo-beneficio del trazado. Asimismo, también sería importante complementar a futuro estos estudios de espacialidad inka con un análisis de la escenificación del planeamiento del sitio, basado en las experiencias y representaciones particulares de las personas dentro del mismo (véase Acuto 2012). En este sentido, y teniendo en cuenta la perspectiva relacional del paisaje planteada en la Introducción, sería interesante explorar otros aspectos que podrían generar cambios visuales que modifiquen las condiciones de visibilidad del sitio, tales como la experiencia del transeúnte según la orientación de su cuerpo durante el trayecto, o según la dirección que adopte hacia o desde un punto determinado del camino.
Otro aspecto a destacar de esta metodología, es que logra disipar la idea de que a través de la simple observación y el sentido común se puede llegar rápidamente a las mismas conclusiones. Al respecto, consideramos que si bien es verdad que a simple vista una persona puede tener una idea general de la visibilidad del sitio, a través de este método podemos obtener un análisis pormenorizado del sitio que a simple vista podría diluirse por diferentes cuestiones ambientales. Además, este método nos permite cuantificar los datos y analizarlos estadísticamente y poder compararlos en un futuro con otros sitios, otros caminos u otras investigaciones.

Para finalizar, creemos que el trazado del Qhapaq Ñan jugó un rol escenográfico en conjunto con otros elementos del sitio, razón por la cual se presentaba como un elemento más de legitimación y poder. Esta situación lo impregnaba de un fuerte significado geográfico y ritual convirtiéndolo en un elemento del paisaje, cuya comprensión iba más allá del simple tránsito.

Agradecimientos A todo nuestro equipo de la División Arqueología del Museo de La Plata y a la comunidad de Londres y Belén de la provincia de Catamarca por su colaboración constante e incondicional durante nuestros trabajos de campo y gabinete. Especialmente expresamos nuestro agradecimiento a la Dra. Aylen Capparelli por su colaboración en la digitalización de la cobertura vegetal utilizada en el SIG; a la Dra. Marina Sardi y al Dr. Iván Pérez por sus comentarios y sugerencias acerca de los análisis estadísticos realizados y a la Lic. Anahí Iácona por sus comentarios generales acerca del manuscrito. También agradecemos al Consejo Nacional de Investigaciones Científicas y Técnicas, Agencia Nacional de Promoción Científica y a la Facultad de Ciencias Naturales y Museo de la Universidad Nacional de La Plata por el apoyo económico brindado durante las investigaciones. Finalmente, agradecemos a los evaluadores de este manuscrito por sus comentarios y observaciones. 


\section{* Referencias Citadas}

ACUTO, F. A. 1999. Paisaje y dominación: la constitución del espacio social en el Imperio Inka. En Sed Non Satiata. Teoría Social en la Arqueología Latinoamericana Contemporánea, A. Zarankin y F. A. Acuto (Eds.), pp. 33-75. Ediciones del Tridente, Buenos Aires.

2012. Landscapes of Inequality, Spectacle and Control: Inka Social Order in Provincial Contexts. Revista Chilena de Antropología 25: 9-64.

ANSCHUETZ, K. F., R. H. WILSHUSEN y C. L. SCHEICK. 2001. An Archaeology of Landscapes: Perspectives and Directions. Journal of Archaeological Research 9 (2): 157-211.

ARANEDA, E. 2002. Uso de Sistemas de Información Geográficos y análisis espacial en Arqueología: Proyecciones y limitaciones. Estudios Atacameños 22: 59-76.

ARCGIS RESOURCES CENTER. 2013. ¿Qué es ModelBuilder? http://resources.arcgis.com/es/help/main/10.1/index. html\#//002w00000001000000 (acceso 29 abril 2014).

BARTONCELLO, F. y L. NUNINGER. 2002. Simulations et modèles d'analyse spatiale. Ager 11:39-46.

BENDER, B. 1993. Introduction: Landscape - Meaning and Action. En Landscape: Politics and Perspectives, B. Bender (Ed.), pp. 1-17. Berg, Oxford.

BERENGUER, J. 2007. El Camino Inka del Alto Loa y la creación del espacio provincial en Atacama. En Producción y circulación prehispánicas de bienes en el sur andino, A. E. Nielsen, M. C. Rivolta, V. Seldes, M. M. Vázquez y P. H. Mercolli (Eds.), pp. 413-443. Editorial Brujas, Córdoba.

BRAY, T. L. 2003. Inka pottery as culinary equipment: food, feasting, and gender in Imperial State design. Latin American Antiquity $14(1): 3-28$.

2004. La alfarería imperial Inka: una comparación entre la cerámica estatal del área de Cuzco y la cerámica de las provincias. Chungara, Revista de Antropología Chilena 36 (2):365-374.

CAPPARELLI, A. 1997. Reconstrucción ambiental de la instalación arqueológica Inka de El Shincal. Tesis Doctoral No 694, Facultad de Ciencias Naturales y Museo, Universidad Nacional de La Plata, La Plata.

COHEN, M. L. 2014. Miradas desde y hacia los lugares de poder. Antofagasta de la Sierra entre 1000 y 1500 años D.C.Arqueología $20(1): 47-72$.
COUSO, M. G., R. A. MORALejO, M. A. GIOVANNETTI, L. M. DEL PAPA, M. C. PÁEZ, J. GIANELLI, L. R. GIAMBELLUCA, M. ARNOSIO y R. A. RAFFINO. 2011. Análisis de la variabilidad material del Recinto 1 - Kancha II: aportes para una comprensión de la política incaica en El Shincal de Quimivil. Arqueología 17: 35-55.

CRIADO BOADO, F. 1991. Construcción social del espacio y reconstrucción arqueológica del paisaje. Boletín de Antropología Americana 24: 5-29.

1993. Visibilidad e interpretación del registro arqueológico. Trabajos de Prehistoria 50:39-56.

1997. Introduction: Combining the different dimensions of cultural space: Is a total archaeology of landscape possible? En Landscape, Archaeology, Heritage, F. Criado Boado y C. Parcero (Eds.), pp. 5-9. Grupo de Investigación en Arqueología del Paisaje, Universidad de Santiago de Compostela, Santiago de Compostela.

D'AlTROY, T. N., A. M. LORANDI, V. WILLIAMS, M. CALDERARI, C. A. HASTORF, E. DEMARRAIS y M. B. HAGSTRUM. 2000. Inka Rule in the Northern Calchaqui Valley, Argentina. Journal of Field Archaeology 27: 1-26.

DE FEO, M. E. y D. GOBBO. 2007. Diseño de un modelo predictivo para la localización de tramos de vialidad incaica mediante la utilización de SIG. En Primer Congreso Argentino de Arqueometría: metodologías cientificas aplicadas al estudio de los bienes culturales, A. Pifferetti y R. Bolmaro (Eds.), pp. 421-428. Humanidades y Artes Ediciones, Rosario, Santa Fe.

DUNCAN, R. B. y K. A. BECKMAN. 2000. The application of GIS predictive site location models within Pennsylvania and West Virginia. En Practical applications of GIS for archaeologist: a predictive modeling kit, K. L. Wescott y R. J. Brandon (Eds.), pp. 37-61. Taylor and Francis, New York.

FARRINGTON, I. S. 1992. Ritual Geography, Settlement Patterns and the Characterization of the Provinces of the Inka Heartland. World Archaeology 23 (3): 368-385.

1998. The concept of Cusco. Tawantinsuyu 5: 53-59.

1999. El Shincal: un Cusco del Kollasuyu. En Actas del XII Congreso Nacional de Arqueología Argentina, C. Diez Marín (Ed.), Tomo I, pp. 53-62. Facultad de Ciencias Naturales y Museo, Universidad Nacional de La Plata, La Plata.

2013. Cusco. Urbanism and Archaeology in the Inka World. University Press of Florida, Florida. 
GARCÍA SANJUÁN, L., S. METCALFE-WOOD, T. RIVERA JIMÉNEZ y D. W. WHEATLEY. 2006. Análisis de pautas de visibilidad en la distribución de monumentos megalíticos de Sierra Morena Occidental. En La aplicación de los SIG en la Arqueología del Paisaje, I. Grau Mira (Ed.), pp. 180-200. Publicaciones de la Universidad de Alicante, San Vicente del Raspeig.

GASPARINI, G. y L. MARGOLIES. 1977. Arquitectura Inka. Centro de Investigaciones Históricas y Estéticas, Facultad de Arquitectura y Urbanismo, Universidad Central de Venezuela, Caracas.

GIL GARCÍA, F. M. 2001. Ideología, poder, territorio. Por un análisis del fenómeno chullpario desde la Arqueología de la Percepción. Revista Española de Antropología Americana 31: 59-96.

GILLINGS, M. y D. WHEATLEY. 2001. Seeing is not believing: unresolved issues in archaeological visibility analysis. En On the good use of Geographic Information Systems in Archaeological Landscape studies, B. Slapsak (Ed.), pp. 25-36. Office for Official Publications of the European Communities (OOPEC), Luxemburgo.

GIOVANNETTI, M., J. SPINA, G. COCHERO, G. CORRADO, L. ALJANATI y M. VALDERRAMA. 2012. Nuevos estudios en el sector "Casa del Kuraka” del sitio El Shincal de Quimivíl (Dpto. Belén, Prov. Catamarca, Argentina). Inka Llaqta 3 (3): 161-19o.

GONZÁLEZ, A. R. 1966. Las ruinas del Shincal. En Actas del Primer Congreso de Historia de Catamarca, tomo III, pp. 15-28. Junta de Estudios Históricos de Catamarca, San Fernando del Valle de Catamarca, Catamarca.

GONZÁLEZ, A. R. 1980. Patrones de asentamiento incaico en una provincia marginal del imperio; implicancias socio-culturales. Relaciones de la Sociedad Argentina de Antropología XIV (1) (NS): $63-82$.

GRAU MIRA, I. 2006. Transformaciones culturales y modelos espaciales. Aproximación SIG a los paisajes de la romanización. En La aplicación de los SIG en la Arqueología del Paisaje, I. Grau Mira (Ed.), pp. 211-226. Publicaciones de la Universidad de Alicante, San Vicente del Raspeig.

GUTIÉRREZ, J. y M. GOULD. 1994. SIG: Sistemas de Información Geográfica. Editorial Síntesis, Madrid.

HYSLOP, J. 1984. The Inka Road System. Institute of Andean Research New York, Academic Press, Orlando.

1990. Inka Settlement Planning. University of Texas Press, Austin.

1992. Qhapaq Ñan. El Sistema Vial Inkaico. Instituto Andino de Estudios Arqueológicos, Petróleos del Perú, Lima.
INGOLD, T. 2000. The Perception of the Environment. Routledge, London and New York.

KVAMME, K. L. 1990. GIS and their effects on regional archeological analysis. En Interpreting Space: GIS and Archeology, K. M. S. Allen, S. W. Green y E. B. W. Zubrow (Eds.), pp. 112-125. Taylor and Francis, New York.

LLOBERA, M. 2003. Extending GIS-based visual analysis: the concept of visualscapes. International Journal of Geographical Information Science 17 (1): 25-48.

2007. Reconstructing visual landscapes. World Archaeology 39 (1): 51-69.

MAKOWSKI, K., M. F. CÓRDOVA, P. HABETLER, M. LIZÁRRAGA. 2005. La plaza y la fiesta: reflexiones acerca de la función de los patios en la arquitectura pública prehispánica de los períodos tardíos. En Boletín de Arqueología PUCP, $\mathrm{n}^{\circ}$ 9, $\mathrm{P}$. Kaulicke y T. D. Dillehay (Eds.), pp. 297-333. Fondo Editorial, Pontificia Universidad Católica del Perú, Lima.

MATOS MENDIETA, R. 1994. Pumpu. Centro Administrativo Inka de la Puna de Junín. Editorial Horizonte, Lima.

MIDDLETON, W. E. K. 1952. Vision through the Atmosphere. University of Toronto Press, Toronto.

MOORE, J. 1996. The Archaeology of Plazas and the Proxemics of Ritual: Three Andean Traditions. American Anthropologist 98 (4): 789-802.

MORALEJO, R. A. 2011. Los Inkas al sur del Valle de Hualfin: organización del espacio desde una perspectiva paisajística. Tesis Doctoral $\mathrm{N}^{\circ} 1150$, Facultad de Ciencias Naturales y Museo, Universidad Nacional de La Plata, La Plata. Disponible en: http://sedici.unlp. edu.ar/handle/10915/5242

2013. La Piedra Hincada de El Shincal de Quimivil. Comechingonia 17 (2): 295-301.

MORALEJO, R. A. y M. G. COUSO. 2012. La organización regional incaica en el Valle de Hualfín (Provincia de Catamarca, Argentina): El Shincal de Quimivíl y la red vial vinculante. En Ciudades y Territorio en América del Sur del siglo XV al XVII, J. F. Buján (Comp.), pp. 77-112. Editorial Nobuko, Buenos Aires.

MORRIS, C. 2013. El palacio, la plaza y la fiesta en el Imperio inca. En Colección Estudios Andinos, no 13, J. R. Topic, J. Pillsbury, H. Lechtman y M. Curatola Petrocchi (Eds.). Fondo Editorial, Pontificia Universidad Católica del Perú, Lima. Institute of Andean Research, New York. 
NIELSEN, A. E. y W. H. WALKER. 1999. Conquista ritual y dominación política en el Tawantinsuyu: el caso de Los Amarillos (Jujuy, Argentina). En Sed Non Satiata. Teoría Social en la Arqueología Latinoamericana Contemporánea, A. Zarankin y F. A. Acuto (Eds.), pp. 153-169. Ediciones del Tridente, Buenos Aires.

RAFFINO, R. A. 1981. Los Inkas del Kollasuyu. Ediciones Ramos Americana, La Plata.

1991. Poblaciones indigenas en Argentina. Urbanismo y proceso social precolombino. Tipográfica Editora Argentina, Buenos Aires.

2004. El Shincal de Quimivíl, R. A. Raffino (Ed.). Editorial Sarquís, San Fernando del Valle de Catamarca.

2007. Poblaciones indígenas en Argentina. Urbanismo y proceso social precolombino. Editorial Emecé, Buenos Aires.

RAFFINO, R. A., A. M. ALBORNOZ, A. L. BUCCI, R. CROWDER, A. IÁCONA, D. E. OLIVERA y G. RAVIÑA. 1978. La ocupación Inka en el N.O. Argentino: actualización y perspectivas. Relaciones de la Sociedad Argentina de Antropología XII (NS): 95-121.

RAFFINO, R. A., R. J. ALVIS, L. N. BALDINI, D. E. OLIVERA y M. G. RAVIÑA. 1982. Hualfín-El Shincal-Watungasta. Tres casos de urbanización Inka en el N.O. argentino. En Actas del LX Congreso Nacional de Arqueología, pp. 470-497. Sociedad Chilena de Arqueología, Museo Arqueológico de La Serena, Chile.

RAFFINO, R. A., R. A. MORALEJO, A. IÁCONA y J. D. GOBBO. 2014. Arquitectura del Paisaje: las Aukaipatas como espacio de poder. En Yachay Wasi. Homenaje a Ian Farrington. School of Archaeology and Anthropology, Australian National University, Canberra.

SOJA, E. W. 1989. Postmodern Geographies: The Reassertion of Space in Critical Social Theory. Verso Press, London.

STEGGERDA, M. 1950. Anthropometry of South American Indians. En Handbook of South American Indians, vol. 6, J. H. Steward (Ed.), pp. 57-69. Bureau of American Ethnology, Bulletin 143, Smithsonian Institution. Government Printing Office, Washington.

THOMAS, J. 2001. Archaeologies of Place and Landscape. En Archaeological Theory Today, I. Hodder (Ed.), pp. 165-186. Polity Press, Cambridge.

VITRY, C. 2000. Aportes para el estudio de caminos incaicos. Tramos Morohuasi - Incahuasi. Salta, Argentina. Editora Gofica, Salta.
WACHTEL, N. 1973. Sociedad e Ideología. Ensayos de historia y antropología andinas. Instituto de Estudios Peruanos, Lima.

WARREN, R. E. 1990. Predictive Modeling of Archaeological Site Location: A Case Study in the Midwest. En Interpreting Space: GIS and Archaeology, K. M. S. Allen, S. W. Green y E. B. W. Zubrow (Eds.), pp. 201-215. Taylor and Francis, New York.

WARREN, R. E. y D. L. ASCH. 2000. A predictive Model of Archeological Site Location in the Eastern Prairie Peninsula. En Practical applications of GIS for archaeologist: a predictive modeling kit, K. L. Wescott y R. J. Brandon (Eds.), pp. 6-36. Taylor and Francis, New York.

WESCOTT, K. L. 2000. Introduction. En Practical applications of GIS for archaeologist: a predictive modeling kit, K. L. Wescott y R. J. Brandon (Eds.), pp. 1-5. Taylor and Francis, New York.

WHEATLEY, D. 1995. Cumulative Viewshed Analysis: a GIS-based method for investigating intervisibility, and its archaeological application. En Archaeology and Geographical Information Systems: A European Perspective, G. Lock y Z. Stancic (Eds.), pp. 171-185. Taylor \& Francis, London.

WHEATLEY, D. y M. GILLINGS. 2000. Vision, perception and GIS: developing enriched approaches to the study of archaeological visibility. En Beyond the Map: archaeology and spatial technologies, G. Lock (Ed.), pp. 1-27. IOS Press, Amsterdam.

2002. Spatial Technology and Archaeology: the archaeological applications of GIS. Taylor \& Francis, New York.

WILliAMS, V., M. P. VILlEGAS, M. S. GHEGGI y M. G. CHAPARRO. 2005. Hospitalidad e intercambio en los valles mesotermales del Noroeste argentino. En Boletín de Arqueología PUCP 9, P. Kaulicke y T. D. Dillehay (Eds.), pp. 335-372. Fondo Editorial, Pontificia Universidad Católica del Perú, Lima.

ZAMORA MERCHÁN, M. 2006. Visibilidad y SIG en arqueología: Mucho más que ceros y unos. En La Aplicación de los SIG en la Arqueología del Paisaje, I. Grau Mira (Ed.), pp. 41-54. Publicaciones de la Universidad de Alicante, San Vicente del Raspeig.

ZAMORA MERCHÁN, M. 2013. Análisis territorial en arqueología: percepción visual y accesibilidad del entorno. Comechingonia 17 (2): 83-106.

ZUIDEMA, R. T. 1995. El sistema de ceques del Cuzco. La organización social de la capital de los Incas. Fondo Editorial, Pontificia Universidad Católica del Perú, Lima. 